De. $0774-9$

ANL/FPP/TM-234

ANL/FPP/TM-234

\title{
INDUCTION FILTERING FOR PROPORTIONAL COUNTER AMPLIFIERS
}

by

E. F. Bennett

MASIER

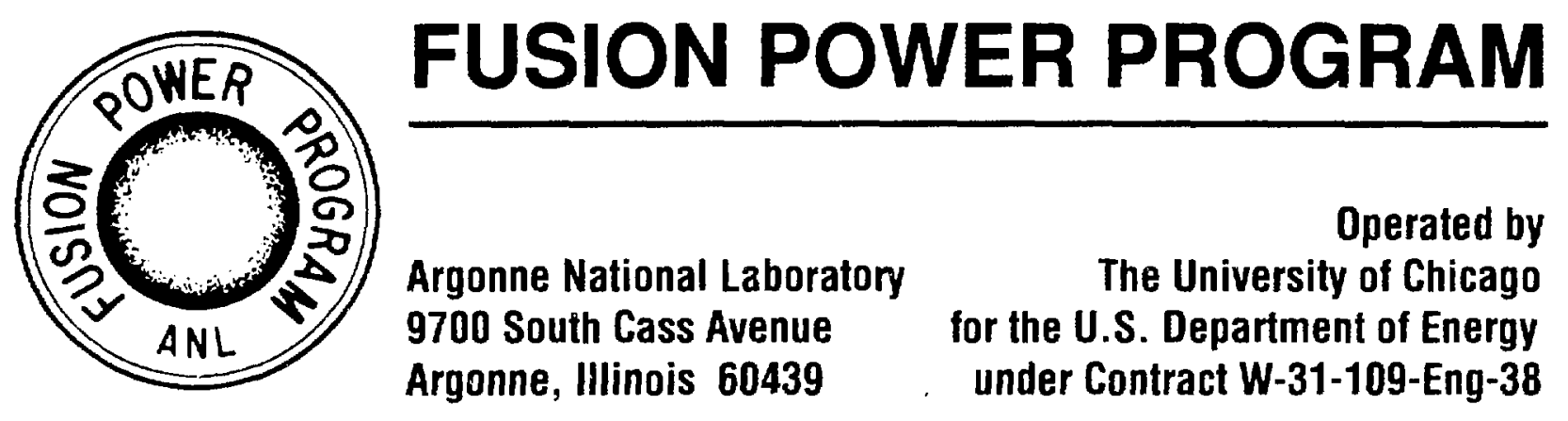




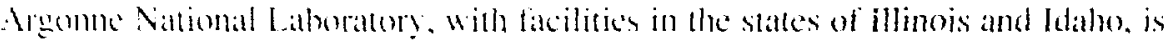
ownd by the linited State government. and operated by The University of Chicago under the provisions of a contrake with the Department of Energy.

\section{DISCLAIMER}

This repon was prepared as an account of work sponsored by an agency of the Linited States Government. Neither the United States Government nor any agency thereot. nor any of their employees. makes any warranty. express or implied. or assumes any legal liability or responsibility for the accuracy. completeness, or usefulness of any information, apparatus, product, or process disclosed. or represents that its use would not infringe privately owned rights. Reference herein to any specific commercial product, process, or service by trade name. trademark. manufacturer. or otherwise, does not necessarily constitute or implv its endorsement, recommendation, or favoring by the L'nited States Government or any agency thereof. The views and opinions of authors expressed herein do not necessarily state or reflect those of the United States Government or any agency thereof.

This report has been reproduced from the best available copy.

Available from the

National Techuical Infomation Service

NTIS Energy Distribution Center

P.O. Box 1300

Oak Ridge, TN 378.31

Price: Printed Copy A0)3

Mierofiche A01 
Distribution Category: Magnetic Fusion Energy (UC-420)

ANL/FPP/TM-- 234

DE89 008992

ANL/FPP/TM-234

ARGONNE NATIONAL LABORATORY

9700 South Cass Avenue

Argonne, Illinois 60439-4801

\section{INDUCTION FILTERING FOR PROPORTIONAL COUNTER AMPLIFIERS}

by

E.F. Bennett

March 1989

Work supported by

Office of Fusion Energy

U.S. Department of Energy

Under Contract W-31-109-Eng-38 
TABLE OF CONTENTS

Page

ABSTRACT $\ldots \ldots \ldots \ldots \ldots \ldots \ldots \ldots \ldots \ldots \ldots \ldots \ldots \ldots \ldots \ldots \ldots \ldots \ldots \ldots$

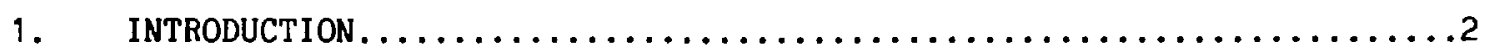

2. PROPORTIONAL COUNTER INDUCTION EFFECT....................

3. PROPORTIONAL COUNTER PULSE AMPLIFIER DESIGN...............

4. AN APPROXIMATE PROPORTIONAL COUNTER INDUCTION FILTER............ 10

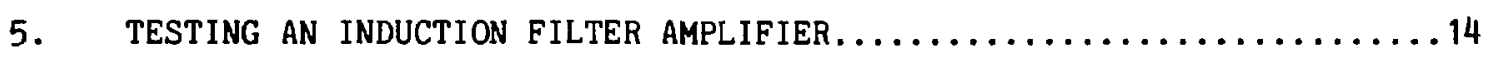

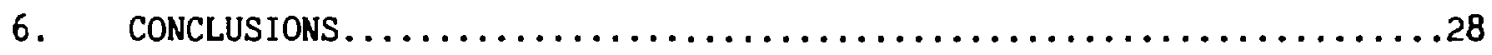

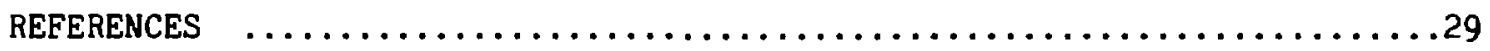




\section{LIST OF FICURES}

Figure No.

Caption

Page

1

Induction profile time dependence

Basic amplifier circuit elements

$3 \quad$ Filter amplifier schematic diagram

4 Overload response comparison, .2 millisec sweep multiplier

5 Overload response comparison, . 1 millisec sweep multiplier

6 Overload response comparison, 50 microsec sweep ulultiplier

Overload response comparison, 20 microsec sweep multiplier

8 Overload response comparison, 10 microsec sweep multiplier

9 Reference amplifier pulse response, low overload counting

10 Filter amplifier pulse response, low overload counting

11 Reference amplifier test pulse spectrum at high overload rates overload rates

\section{LIST OF TABLES}

Table No.

\section{Caption}




\title{
INDUCTION FILTERING FOR \\ PROPORTIONAL COUNTER AMPLIFIERS
}

\author{
E.F. Bennett
}

\section{ABSTRACT}

The interesting range of the neutron spectrum in fusion blankets includes all energies up to $14 \mathrm{Mev}$. A limited region of this spectrum (from $1 \mathrm{Kev}$ to 1 Mev) is amenable to recoil-proton proportional counting and these measurements are now made routinely at the FNS facility at JAERI. One aspect of proportional counter measurements is a consequence of the necessity to acquire data at the lower energies in the presence of much more numerous events of higher energy which overload and saturate pulse amplifiers. Saturation, together with high count rates required for good statistical measurement precision, can introduce systematic errors into the determination of proton recoil distributions. Here we treat this overload distortion problem by a filtering technique designed specifically for the unique ionization response of the proportional counter. Amplifiers having optimal filtering will provide measured proton recoil spectra with substantially less distortion than amplifiers optimized for other types of detectors. 


\section{INTRODUCTION}

Proportional counters are frequently required to operate at high count rates and also with high overload rates. Gas multiplication in a proportional counter may be increased almost arbitrarily (ultimately limited by spacecharge saturation) to allow detection of even very weakly ionizing events including events which produce only a single free electron. In the process of measurement at the lowest energies, heavy overloading of amplifier systems may occur from events across the spectrum of events with energies above those of interest. This is a common radiation detection problem, but some exceptionally severe distortion can occur for the proportional counter due to the inherent induction response to free electrons created in the gas. The problems are much worse for cylindrical proportional counters than for parallel plate detectors (which include solid state as well as gas-filled devices). Parallel plate detectors possess an electric field of nearly constant magnitude across the full sensitive volume and this allows rapid charge collection. Where charge collection times are not in excess of the shapiag time of the data pulse, pileup and overload distortion can be readily held to low levels for most practical situations.

The proportional counter induction effect arises from the circumstance that the anode consists of wire of very small diameter required to achieve an electron avalanche close to the anode. Electric fields, though strong near the wire where avalanche occurs, become quite weak at large distances and it may require a millisecond or more to collect at the cathode all of the positive ions created in the avalanche. If one imposed count rate limitations upon proportional counters that assured low probability for multiple events occurring within the full induction time response, these counters would be severely rate limited and essentially worthless as radiation detectors. One is left, therefore, with the necessity of minimizing the impact of the slow component of the induction effect in order to operate at acceptably high count rates without compromising spectroscopic quality.

Larson and Powell ${ }^{(1)}$ have addressed some of these considerations previously, and they indicate advantages accompanying the use of amplifiers with baseline restoration for proportional counters. Most commercia:ly available amplifiers include a baseline restorer. 
In the following report, filter modifications to conventional pulse amplifiers are discussed which can also produce very useful improvements in the quality of spectroscopy using proportional counters. The use of filtering, as will be described here, is an alternative (or an addition to) a baseline restorer and is superior to some, and probably all, of the baseline restorers in current usage. A disadvantage of the filtering described here is that tuning is required which is specific for the gas type and pressure and for the anode/cathode configuration of the counter. Retuning is required where changes in any of these basic counter parameters are made. No general purpose amplifier exists for use with proportional counters; each counter requires an amplifier with parameters specific to details of the counter design.

\section{PROPORTIONAL COUNTER INDUCTION EFFECT}

The standard configuration for a proportional counter is one of concentric cylinders; the inner cylinder (anode) consisting of a wire of small diameter, $d_{a}$, and an outer cylinder (cathode) consisting of a tube of diameter, $d_{c}, 1000$ times or so greater than $d_{a}$. The counter is filled with a suitable gas in which ionization occurs from either an external or an internal radiation source. Since the anode is positively biased relative to the cathode, free electrons created by an ionizing event are swept inward along radially symmetric electric field lines until they are collected on the anode.

The counter is operated at some combination of gas pressure and voltage that allows electron multiplication to occur in the region of high electric field close to the anode wire. Large numbers of secondaries may be produced per primary electron and the resulting pulse, while remaining proportional in amplitude to the initial electron number, is increased sufficiently to allow measurement well in excess of system noise, even for a single initiating electron.

The electrostatics of pulse formation in the proportional counter is discussed in detail by Wilkinson (2), for example. The induction profile for proportional counters contrasts markedly with that for devices using semiconductors or gases in essentially parallel plate geometry. The induction effect of the initia: electrons can be ignored altogether in the proportional counter; one need only be concerned with movement of the residue of positive 
ions created in the anode avalanche. This is in consequence of the fact that gas multiplication can occur only in the high field region close to the anode wire. Because the electrons formed in the avalanche are collected at the anode immediately, the electric field does negligible work upon them. Since the mobility of positive ions is orders of magnitude less than for electrons at the same field/pressure ratio, collection of positive ions which must occur to terminate the pulse can easily extend to a millisecond or more depending upon details of the counter and the filling gas.

The long collection time for positive ions in proportional counters is a consequence of the very weak electric field (which varies inversely as the distance from the anode center) over most of the anode-cathode distance. Assuming that the mobility of positive ions is proportional to the electric field, the induction voltage initiated by a single collected electron of charge e can be shown to have the form

$$
I(t)=-\left(e /\left(2 c_{d} \log \left(d_{c} / d_{a}\right)\right) \log (t / h+1)\right.
$$

for times less than

$$
t_{\max }=h\left(d_{c}^{2} / d_{a}^{2}-1\right)
$$

after which $I(t)$ remains constant.

The characteristics time constant $h$ can be expressed in terms of anode and cathode radii, counter voltage $v$, and positive ion mobility $k$ as:

$$
h=d_{a}^{2} \log \left(d_{c} / d_{a}\right) /(8 \vee K)
$$

The positive ion collection time ( $t_{\max }$ in Eq. 2) marks the end of the event. After $t_{\max }$ the induction effect ceases to increase and remains at the value $-e / C_{d}$ where $C_{d}$ is the total anode capacitance of the counter.

Typically, for a counter having a cathode to anoid ratio of 1000 , and with several kilovolts bias voltage, the magnitude of the characteristic ion mobility time $h$ will be about one nanosecond which, through Eq. 2, will result in a collection time of about one millisecond. While the total collection time may be quite long, it is also true that most of the induction effect is 
realized in the rather short time interval of a few microseconds suitable for pulse amplitude spectroscopy at relatively high count rates. If a value for $h$ of one nanosecond is assumed, Eq. 1 predicts that $60 \%$ of the entire induction effect will be realized within the first 10 microseconds. Pulse amplifiers with a primary time constant of a few microseconds will respond to most of the induction effect within the primary "data" time interval.

That portion of the induction effect which continues on until positive ion collection can be very troublesome however, given the existence of high count rate and overload; conditions which are relatively common in many applications for these counters.

Figure 1 contains a plot of the quantity

$$
\begin{array}{ll}
U(t)=\log (t / h+1) & t<t_{\max } \\
U(t)=2 \log \left(d_{c} / d_{a}\right) & t>t_{\max }
\end{array}
$$

which differs from Eq. 1 only by a normalization factor, and where $h$ is set equal to 1.1 nanoseconds. Assuming a cathode/anode ratio of 1000 , collection of positive ions occurs at $1.1 \mathrm{millisecond;}$ at this time the induction profile (solid line) ceases to change and is at its maximum value. The origin of the time axis is set to 10 microseconds; prior to this, the induction profile rises very steeply from its value of zero at $t=0$. Any amplifier used to shape and increase the magnitude of the fast (less than 10 microsecond) component of the induction effect should also minimize the long-time component of the induction in order to maintain overload and pileup effects at the minimum possible level.

\section{PROPORTIONAL COUNTER PULSE AMPLIFIER DESIGN}

The design of amplifiers for radiation detectors has been extensively discussed in the literature (Refs. 1, 2, and 3 for example). No single combination of parameters for an amplifier can be expected to be optimal for all types of detectors under all circumstances of operation. A single time constant may be preferred at high count rates due to the shorter overall pulse duration. Double-differentiation may be acceptable under some conditions of count rate and overload. Invariably, one would like to minimize random noise. Random noise should all originate in the preamplifier (for a well- 
ordered counting system) but pulse shaping circuits in the main amplifier will modify whatever noise is present at the input. Multiple integration (Gaussian approximation) will reduce noise levels but at the expense of a wider filse at baseline and a peak that occurs later in time. Baseline restorers may be added to the output stage. These will increase noise but provide improved baseline stabilizy.

All amplifiers include a preamplifier pole-zero at the input. This allows the use of long time constants and high values of resistance in the preamplifier feedback $R C$, a condition necessary to minimize thermal resistance noise. The amplifier input pole-zero provides a single time constant (of microsecond duration) for the data pulse while removing the long preamp feedback RC. If the induction effect is well approximated by a "step" function in time there will be no additional time constants following the input pole zero filter.

This last point is an important difference between amplifiers for proportional counters and for detectors with plate geometries having high electron/ion (or electron/hole) mobilities. The proportional counter induction effect (see Fig. 1) continues to change far beyond the few microseconds required for amplitude analysis and there is, consequently, a complicated residual time dependence of the amplifier pulse.

Ideally, one would like to introduce a network into the amplifier which filters out the slow induction effect, whatever its characteristics, leaving only a single short time-constant pulse at the output for analysis. It is clear how to proceed in principle. Figure 2 contains two elements of the electronics system, the preamplifier and the pole-zero filter, together with their respective transfer functions in the usual Laplace representation. $I(t)$ from Eq. 1 is the voltage source input. $T$ is the preamplifier time constant $\left(T=R_{F} C_{F}\right.$ ) and $C_{d}$ is the same detector anode capacitance as appears in $E q$. 1. The gain, A, of the inverting amplifier in Fig. 2-a is assumed very large and its input is assumed to draw no current. The transfer function for the preamp, $\overrightarrow{\mathrm{PA}}(\mathrm{s})$, is readily derived from these parameters and is given in $\mathrm{Fig}$. 2-a. Impedance of the voltage source driver for the pole-zero section of Fig. 2-b is assumed to be small while impedance of the buffer at the output of the pole-zero filter is assumed to be large. With these assumptions, the transfer function of the pole-zers section, $\overline{\mathrm{PZ}}(\mathrm{s})$, is given in Fig. 2-b. 


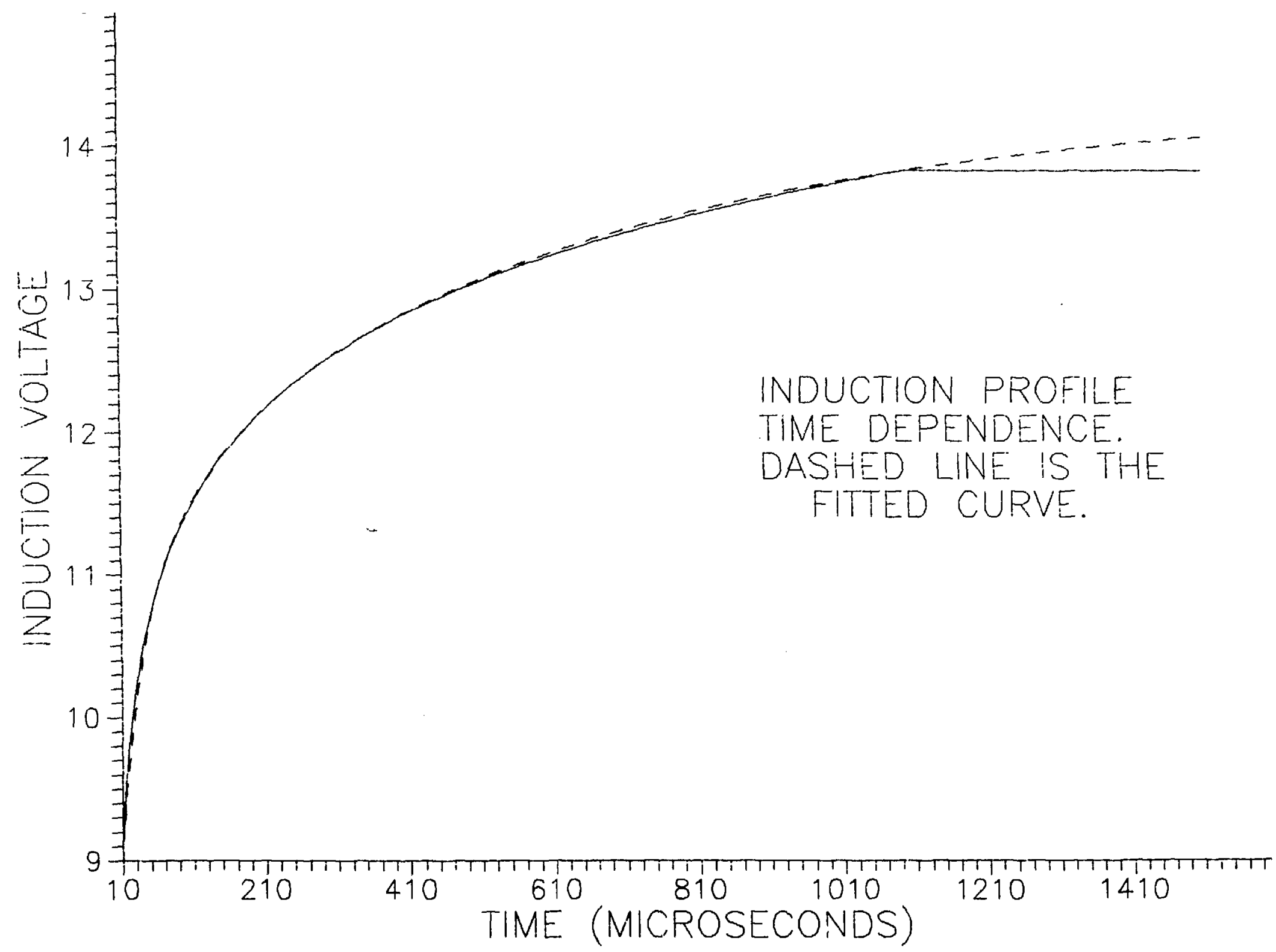

Fig.1 Induction Profile Time Dependence. 
A: PREAMPLIFIER

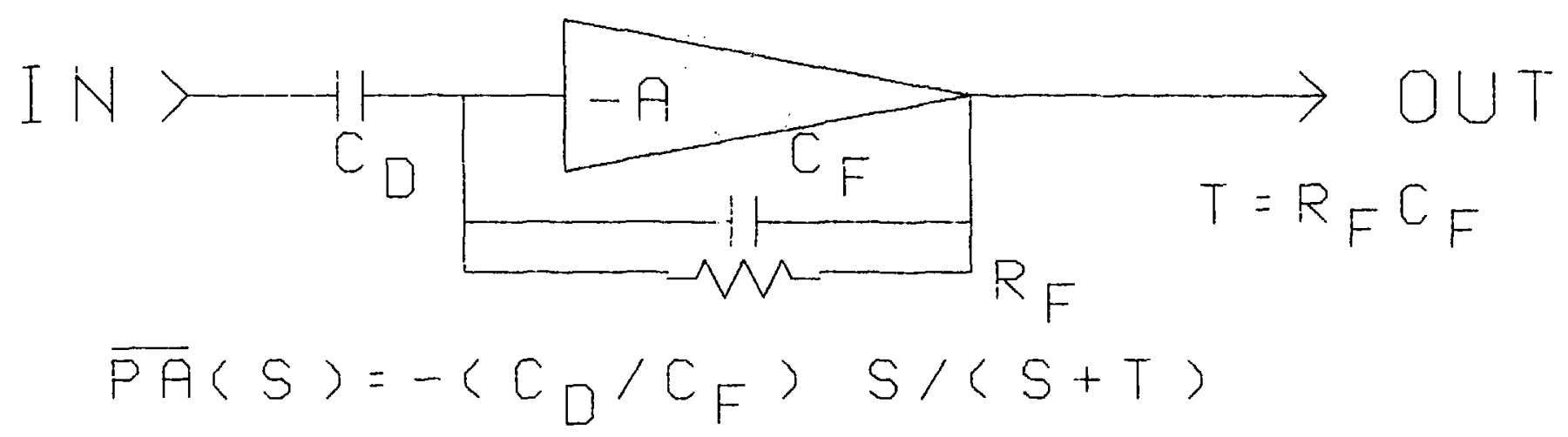

$\infty$

B: POLE ZERO FILTER

$I N>\sum_{\sum_{R}} \rightarrow O U T$

$P O L E=\left(R+R^{\prime}\right) /\left(R C R^{\prime}\right)$

ZERO = $1 / R C$

$\overline{P Z}(S)=(S+Z E R O) /(S+P O L E)$

Fig.2 Basic Amplifier Circuit Elements. 
If we define $\bar{I}(s)$ to be the transform of $I(t)$ in Eq. 1 , and if we allow $W(s)$ to represent some as yet unspecified filter in the main amplifier which is inserted following the pole-zero at the input, ther the transform of the output of this sequence will be the product of the following terms:

(1) $\bar{I}(s)$...the induction effect transform

(2) $\overline{P A}(s) \ldots$ the preamplifier response

(3) $\overline{P Z}(s)$...the preamplifier pole zero

(4) W(s)...the main amplifier filter

Since we wish to retain only a data pulse having a single time constant $q$ at the output, we set the product of the four terms above equal to the transform of the data pulse:

\section{$\mathrm{G} /(\mathrm{s}+\mathrm{q})$ (transform of Ge-qt)}

where $G$ is an arbitrary gain constant.

The resulting equality is, therefore,

$$
\bar{I}(s) \overline{P A}(s) \overline{P Z}(s) W(s)=G /(s+q)
$$

For purposes of filter design, we do not need to retain constant "gain" factors. The overall amplification of the system is important, of course, but its absolute value will not influence the design of a filter. Also, note that the absolute induction effect $I(t)$ in Eq. 1 is of negative magnitude but the preamplifier is inverting and consequently the product of $\overline{\mathrm{I}}(s)$ and $\overline{\mathrm{PA}}(s)$ is positive. We can, therefore, use the un-normalized induction $U(t)$ as $g$ iven in Eq. 4 (and plotted in Fig. 1) with positive sign if we also let $\overline{P A}(s)$ for the preamplifier also be of positive sign. If transforms for the elements in Fig. 2 are now introduced into $\mathrm{Eq} .5$ and we ignore the fixed gain $\tilde{s}$, the result is:

$$
\bar{U}(s)[s /(s+T)][(s+T) /(s+q)] W(s)=1 /(s+q)
$$


from which $w(s)$ is found to be

$$
W(s)=1 /(s \bar{U}(s)) \text {. }
$$

The actual sequence in which filters appear in the amplifier is irrelevant in the absence of overload pulses. Where overload occurs, however, it is important to remove terms of large amplitude in the early stages of the filter before substantial amplifier gain is realized.

It is always desirable to introduce the preamplifier pole-zero filter at the in amplifier input. The order in which additional filters are introduced should then give priority to the terms with largest amplitude. In this way, overloading which occurs is limited to the duration of the data pulse, which has the shortest time constant of the system. A fundamental assumption in handling overload is that the preamplifier never saturates. If preamplifier saturation occurs, the system will not only be dead for long periods of time, but spurious pulses may be produced during recovery.

Equation 7 defines the characteristics of the ideal proportional counter filter, with $\bar{U}(s)$ given by the transform of Eq. 4. If this filter were realized exactly, its introduction into the amplifier (following the preamplifier pole-zero filter) would result in a single "data" pulse of short time constant followed by an unchanging baseline. Pileup and overload distortion would be reduced to the absolute minimum. The pulse amplifier could be further modified to include one or more integrations for noise reduction as well as a baseline restorer.

If the induction effect, $U(t)$, can be taken as a simple "step" function, the transform $\bar{U}(s)$ will be proportional to $1 / \mathrm{s}$ and $E q$. 7 would then reduce to $W(s)=1$ (apart from an uninteresting gain factor). For the ideal voltage step induction, no filtering beyond the preamplifier pole-zero is required.

\section{AN APPROXIMATE PROPORTIONAL COUNTER INDUCTION FILTER}

The ideal filter for the proportional counter is defined by Eqs. 4 and 7. If the transform $\bar{U}(s)$ could be expressed as a ratio of polynomials, $W(s)$ could be implemented as a series of simple pole-zero elements as defined in Fig. 2-b (Ref. 5 for example). It is apparent, however, that $\bar{U}(s)$ cannot be expressed exactly in this simple form since the induction function has 
discontinuous derivatives at the ion collection time (after which the induction function remains constant). Although an exact solution may not be possible, an approximation to the induction function can be made through use of the approximate expression

$$
U(t)=a_{0}+\sum_{n=1}^{N} a_{n}\left(1-e-\lambda_{n} t\right) \quad t<t_{\max } .
$$

The approximate expression of Eq. 8 will agree (through a suitable choice of the parameters $a_{0}, a_{n}, \lambda_{n}$ and $N$ ) quite well with the true induction effect over the time interval up to collection time. Systematic error between the true induction and the approximation of Eq. 8 will onset at collection time. The discrepancy between the true induction and the approximation will be relatively small however (Fig. 1) even after positive ion collection time, and the associated systematic error will be much less than the total slow component of the induction.

The "step" component $a_{0}$ in the approximation of Eq. 8 may be chosen rather arbitrarily to contain the entire induction for times less than a few microseconds or so. The very rapid increase in the induction for times less than the data pulse decay time constant of a few microseconds will allow this "step" approximation to be made with little attendant error. After removal of the step component, the remaining induction profile was fit using a Variable Metric Minimization (VMM) procedure (Ref. 6) in which a "best fit" set of parameters $\mathrm{a}, \lambda$ is defined for any order $\mathrm{N}$ according to Eq. 8. In principle, $N$ is arbitrary in the VMM procedure. In practice, if $\mathrm{N}$ is large, convergence may be slow and the result may contain values for $\lambda$ which are close together. By varying the order $N$, it was found that an $N$ of at least three was required for close approximation; an $\mathrm{N}$ of four lead to only minimal improvement in the fit and more than four terms produced convergence difficulties with the VMM procedure.

Figure 1, as an example, contains both the induction effect (Eq. 4, plotted as a solid line) and the third order VMM fit (plotted as a dashed line). Results are plotted only for values of induction greater than 10 microseconds; below this value the induction may all be lumped into the "step" component $a_{0}$ of Eq. 8. As can be seen, the true induction and its fitted approximation are almost indistinguishable from each other (everywhere less 
than a $1 \%$ disagreement) until collection time, which was taken as 1.1 milliseconds. This value for collection time is consistent with the value observed for a counter used for overload testing experiments described in Section 5. After collection time, the fitted profile diverges from the true value but not by a large amount. Table 1 contains the set of coefficients of Eq. 8 derived from the fit to the induction effect $U(t)$ given by Eq. 4. Although the particular induction profile selected for analysis was chosen arbitrarily (corresponding to a collection time of 1.1 milliseconds), an induction profile for any other collection time in the range of interest for proportional counters can be fit equally well with three or four terms in an expansion as given by $\mathrm{Eq} .8$.

Table 1.

Parametric Fit of an Induction Time Profile

Ion Collection Time $=1.1 \mathrm{Milliseconds}$

$b / a=1000$

Amplitude

Time Constant $1 / \lambda$

(Microseconds)

\begin{tabular}{lll}
\hline $\mathrm{a}_{0}$ & 7.7830 & - \\
$\mathrm{a}_{1}$ & 2.7606 & 634.8 \\
$\mathrm{a}_{2}$ & 1.3255 & 98.46 \\
$\mathrm{a}_{3}$ & 1.9471 & 24.43 \\
\hline
\end{tabular}

If we use for the induction profile the expression in Eq. 8, we can establish the filter parameters without difficulty. The transform of Eq. 8 is

$$
U(s)=a_{0} / s-\sum_{n=1}^{3} a_{n} /\left(s+\lambda_{n}\right)
$$

Eq. 9 is readily expressed as a polynomial ratio of the form

$$
s \bar{U}(s)=P(s) / Z(s)
$$


$Z(s)$ is already in factored form and may be written as

$$
Z(s)=\left(s+\lambda_{1}\right)\left(s+\lambda_{2}\right)\left(s+\lambda_{3}\right)
$$

while $P(s)$ may be expanded as

$$
\begin{aligned}
& P(s)=a_{0} s^{3}+\left[a_{0} \Sigma \lambda_{i}+\Sigma a_{i} \lambda_{i}\right] s^{2}+\left[\lambda_{2} \lambda_{3}\left(1-a_{1}\right)+\right. \\
& \left.\lambda_{1} \lambda_{3}\left(1-a_{2}\right)+\lambda_{1} \lambda_{2}\left(1-a_{3}\right)\right] s+\lambda_{1} \lambda_{2} \lambda_{3} .
\end{aligned}
$$

The polynomial expansion of $\mathrm{Eq} .12$ for $\mathrm{P}(\mathrm{s})$ may be factored (using a standard numerical procedure) into the product of terms $s+p$, where $p$ are the roots of Eq. 12, giving the result

$$
P(s)=a_{0}\left(s+p_{1}\right)\left(s+p_{2}\right)\left(s+p_{3}\right)
$$

Values for the roots $p$ (poles) of $\mathrm{Eq} .12$ together with the (zeros) for the example shown in Fig. 1 are provided in Table 2.

Table 2.

Poles and Zeros for the Filter Sections

\section{Filter}

Section 1/2ero

(Microseconds)
1/Pole

(Microseconds)

634.8

98.46

24.43

511.6

86.99

19.33

The filter function $W(s)$ defined in $\mathrm{Eq} .7$ may now be expressed as

$$
w(s)=\left[\left(s+\lambda_{1}\right) /\left(s+p_{1}\right)\right]\left[\left(s+\lambda_{2}\right) /\left(s+p_{2}\right)\right]\left[\left(s+\lambda_{3}\right) /\left(s+p_{3}\right)\right] .
$$

$W(s)$ is the product of three terms of the pole-zero type and may be easily realized by a buffered sequence of three of the simple circuit elements shown in Fig. 2-b. From the poles and zeros in Table 2, suitable values for the two resistors and the capacitor can be derived for each section using the formula 
in Fig. 2-b. The only free parameter in the induction profile (apart from gain terms which we ignore) is the ion collection time. This was chosen to be $1.1 \mathrm{milliseconds}$ for the profile of Fig. 1, which lead to the filter system of Eg. 14 with poles and zeros given in Table 2. Another choice of positive ion collection time will produce a different set of fitted parameters $a, \lambda$. The filter must, therefore, be "tuned" to the proportional counter with which it is to be used. There is no universal filter solution, although three filter sections are probably sufficient for any value of the characteristic positive ion mobility time.

The positive ion mobility time $h$, as defined by $\mathrm{Eq} .3$, determines the collection time and is dependent upon counter voltage. This is not a very serious complication since one will be primarily concerned with the effectiveness of the filter system at the highest range of voltages over which the counter is used. The lower voltages are accompanied by much less overload and pileup and filter settings become much less critical. In addition, the total range of voltage over which counters operate is seldom more than about $25 \%$ or so of the maximum value of voltage.

\section{TESTING AN INDUCTION FILTER AMPLIFIER}

An amplifier, which included a three-section filter as described in Section 4, and which will be referred to subsequently as the Filter Amplifier, was constructed and compared with an amplifier of commercial design (designated subsequently as the Reference Amplifier) which did not contain provision for filtering of the slow induction component. The commercially available amplifier was a NIM module which the author has used for about seven years for pulse counting experiments. The data pulse was set at two microseconds. Double integration was used to produce a fairly good "Gaussian" approximation and the output stage included a baseline restorer. It is the author's opinion that this amplifier is typical of NIM modules offered by several U.S. manufacturers during the late 1970's.

The Filter Amplifier schematic is shown in Fig. 3. Gain was achieved by the buffer amplifiers which isolate each filter section and provide a high input and a low output impedance. The data pulse was set at 2 microseconds. A single active integrator ( 2 microseconds) was inserted before the output driver. No baseline restorer was included. DC coupling was maintained 


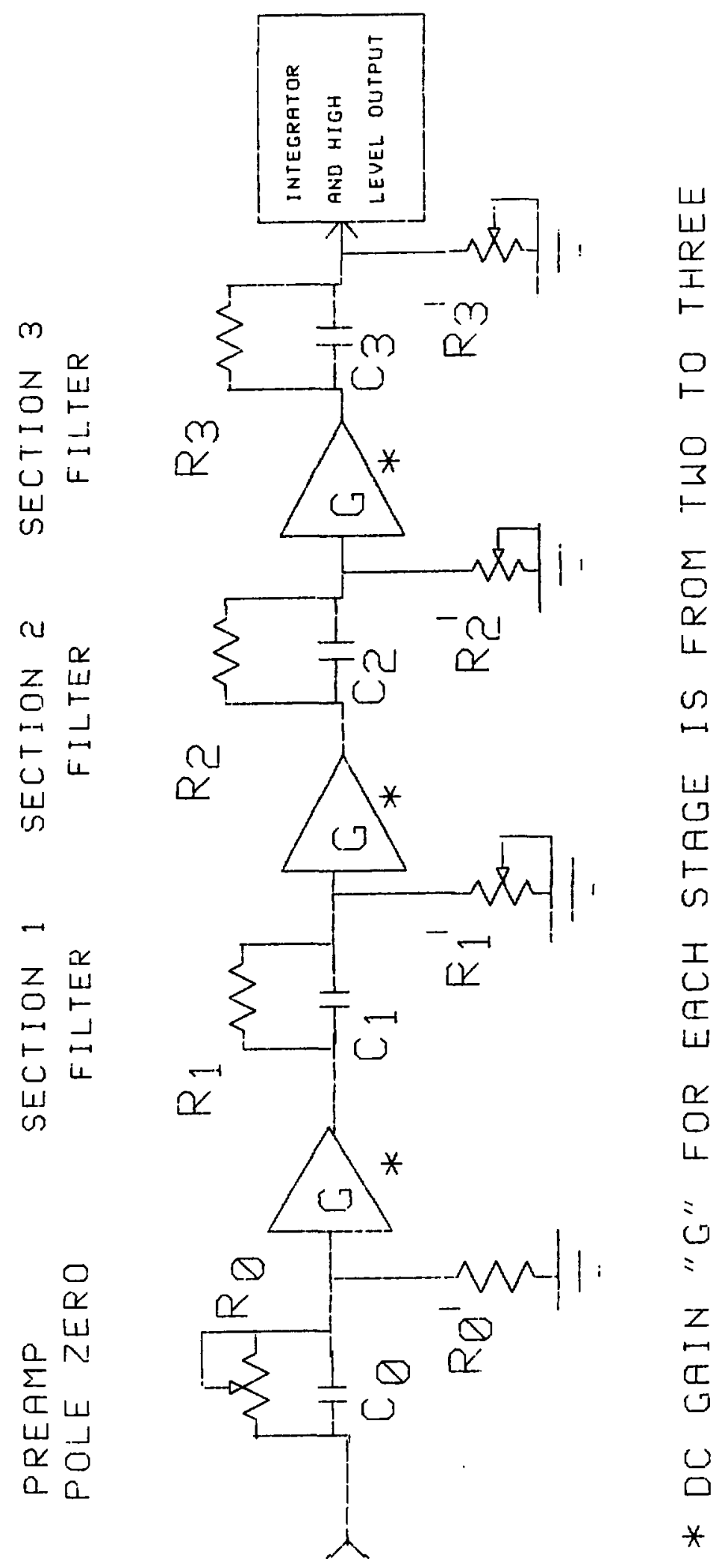

dWHayd WOVd I INANI 
throughout; the output baseline level could be set by an adjustable compensation voltage mixed in before the output stage.

Both the Reference Amplifier and the Filter Amplifier had preamplifier pole-zero cancellation filters at their inputs. Although this adjustment can be set well enough for semiconductor detectors by observing radiation pulses, it is not possible to make an adequate adjustment for proportional counters due to the presence of the slow induction effect, which is the subject of this report. Instead of radiation pulses, a high quality voltage step function was input through the preamplifier, and the pole-zero adjustments were made to remove the long "tail" (250 microseconds) due to the feedback time constant. The same procedure was used for both the Filter Amplifier and the Reference Amplifier. For this adjustment on the Filter Amplifier, the compensation was observed at the output of the first buffer stage prior to any supplemental filtering. The preamplifier was a design (Ref. 7) that permitted the output to reach higher values of voltage than is normally allowed before saturation occurs.

The counter used for these tests had a cathode diameter of one inch and an anode diameter of one $\mathrm{mil}$ (cathode/anode ratio of 1000). It was filled with about 6 atmospheres of hydrogen gas and also contained small amounts of methane and of nitrogen. The methane was needed for quenching and the nitrogen for calibration. The counter was exposed to neutrons from an $\mathrm{Am}-\mathrm{Be}$ source. Both source and counter were enclosed within a small pile consisting mostly of iron and lead. The ionizing events in the counter were primarily those from proton recoils in the hydrogen gas (some gamma background was also present) and the maximum energy deposited was about $3 \mathrm{MeV}$.

In order to do a direct comparison of the two amplifiers under identical conditions, pulses from the preamplifier were fed to both in parallel and also to a third amplifier used for gating. The gains (peak channel of a test pulser input to the preamp) were set equal for the two amplifiers; the absolute gain was chosen consistent with requirements for neutron spectroscopy measurements.

A dual-trace storage scope was used for testing; the Reference Amplifier and the Filter Amplifier were both used as input and the trace was set to display both alternatively. A gate for the scope was generated using a window discriminator on the output of the third amplifier, whose gain was set very 
low. In this configuration, a gate for the scope was generated for pulses which were 160 times overloaded. This is not an extreme overload situation but does correspond to conditions of practical interest where measurements are required below about $10 \mathrm{KeV}$ in the presence of a broad neutron-induced ionization spectrum extending to $1 \mathrm{MeV}$ or so. The vertical gain (volts/cm) for both storage scope channels was set to the same value. DC level output for each amplifier channel was set to provide an identical DC baseline level in the absence of ionization pulses. The baseline level will be reached at an asymptotically long time after any single event (assuming that additional events do not occur in the interval) for either amplifier. The total detection rate was limited to a very low value by placing the detector at a distance from the "source. This was necessary to avoid the recording of multiple events within the rather long time interval of a few milliseconds required for a complete pulse profile observation. A filter parameter set taken from the fitting procedure described for the induction profile and listed in Table 2 gave fair compensation assuming positive ion collection occurred at $1.1 \mathrm{milliseconds.} \mathrm{A} \mathrm{better} \mathrm{set} \mathrm{of} \mathrm{resistance/capacitance} \mathrm{values}$ was obtained by varying components of the filter sections while observing the single-event response profile. Using three well-separated time constants, iteration to a fairly good set of values could be achieved rather quickly. Good filter solutions could be found using a range of capacitance values (Fig. 2-b) together with optimizing adjustments of the related resistors to produce associated pole/zero pairs. There is no universal significance attached to the filter settings; they will depend upon the counter filling gas and pressure as well as upon anode and cathode dimensions. A different filter will be required whenever changes in the detector are made.

A comparison of the single-event response of the two amplifiers is shown in Figs. 4 through 8 . The output of the Eilter Amplifier saturated at a somewhat higher voltage than did the output for the Reference Amplifier. Apart from this, the higher voltage trace was from the Reference Amplifier in each of these figures. The horizontal (sweep) axis is absolute. The time of initiation of the pulse is the left-most origin of the dual scope trace. The counter bias voltage was maintained at 4500 volts. Figure 4 is perhaps the most interesting of the sequence since it includes the positive ion collection time "blip" at about $1.1 \mathrm{milliseconds} \mathrm{(collection} \mathrm{time} \mathrm{is} \mathrm{specific} \mathrm{for} \mathrm{each}$ proportional counter depending upon gas, pressure and anode/cathode 


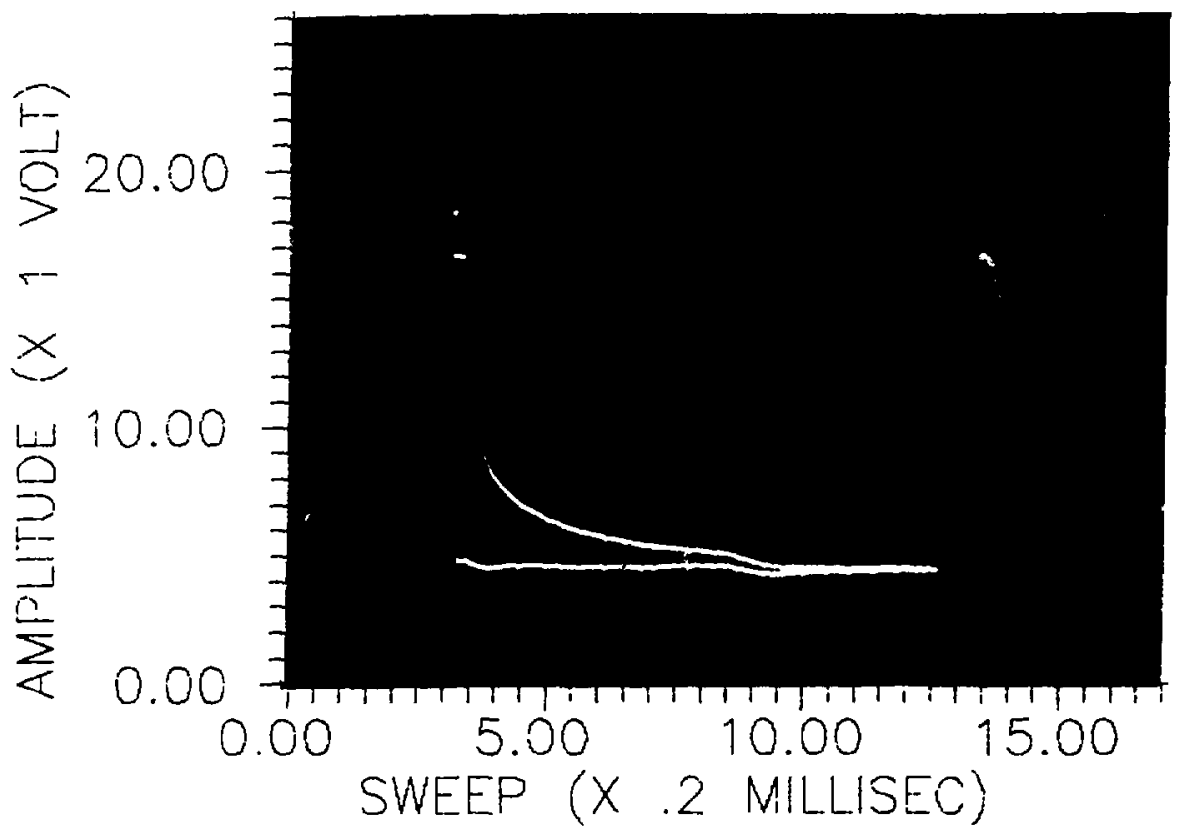

Fig.4 Overload Response Comparison, .2 Millisec Sweep Multiplier. 


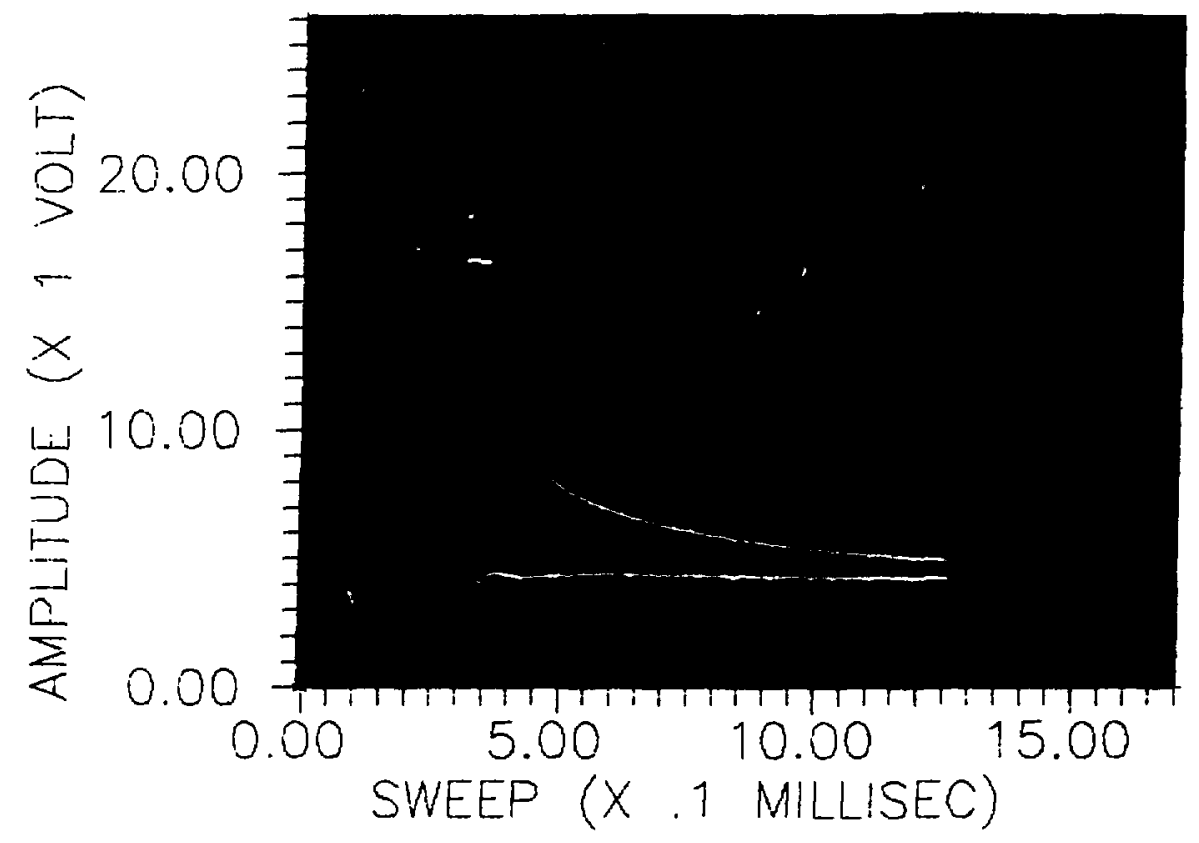

Fig. 5 Overload Response Comparison, . 1 Militisec Sweep Multiplier. 


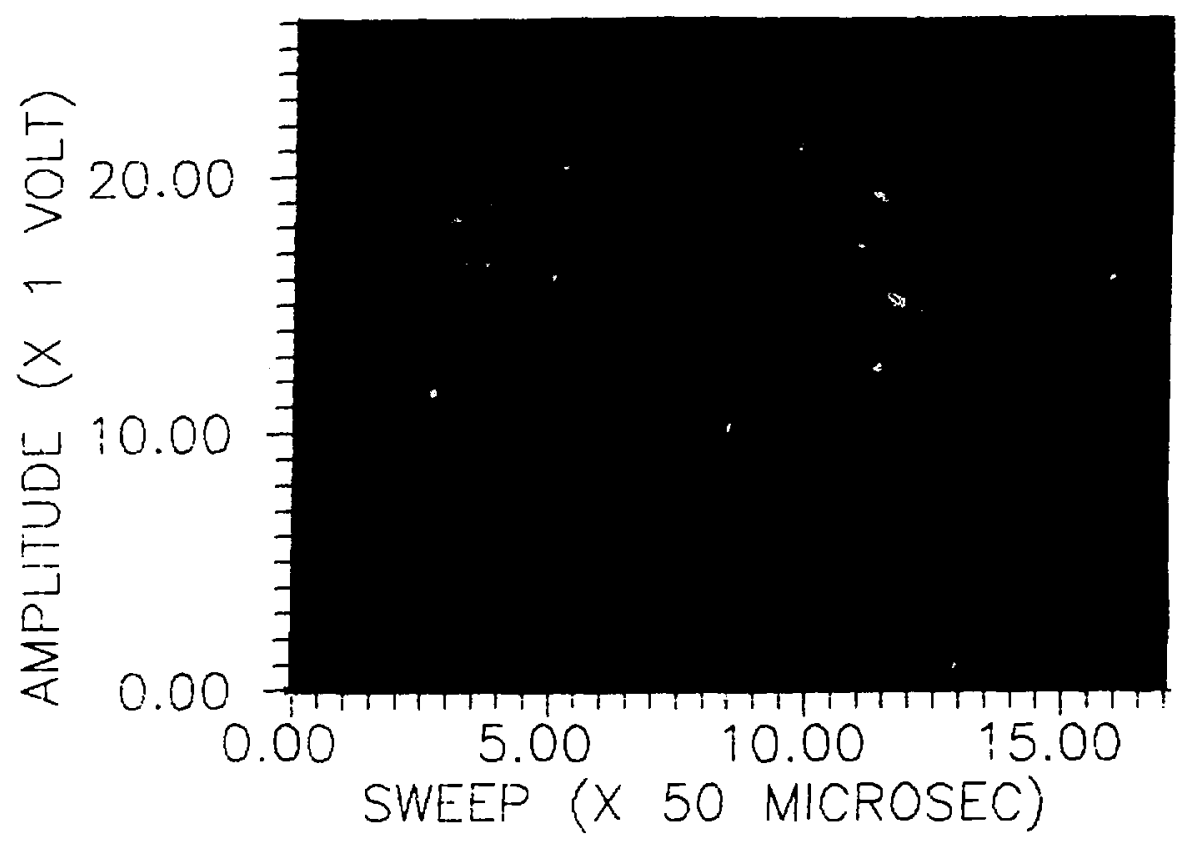

Fig. 8

Over load Response Comparison, 50 Microsec Sweep Multiplier. 


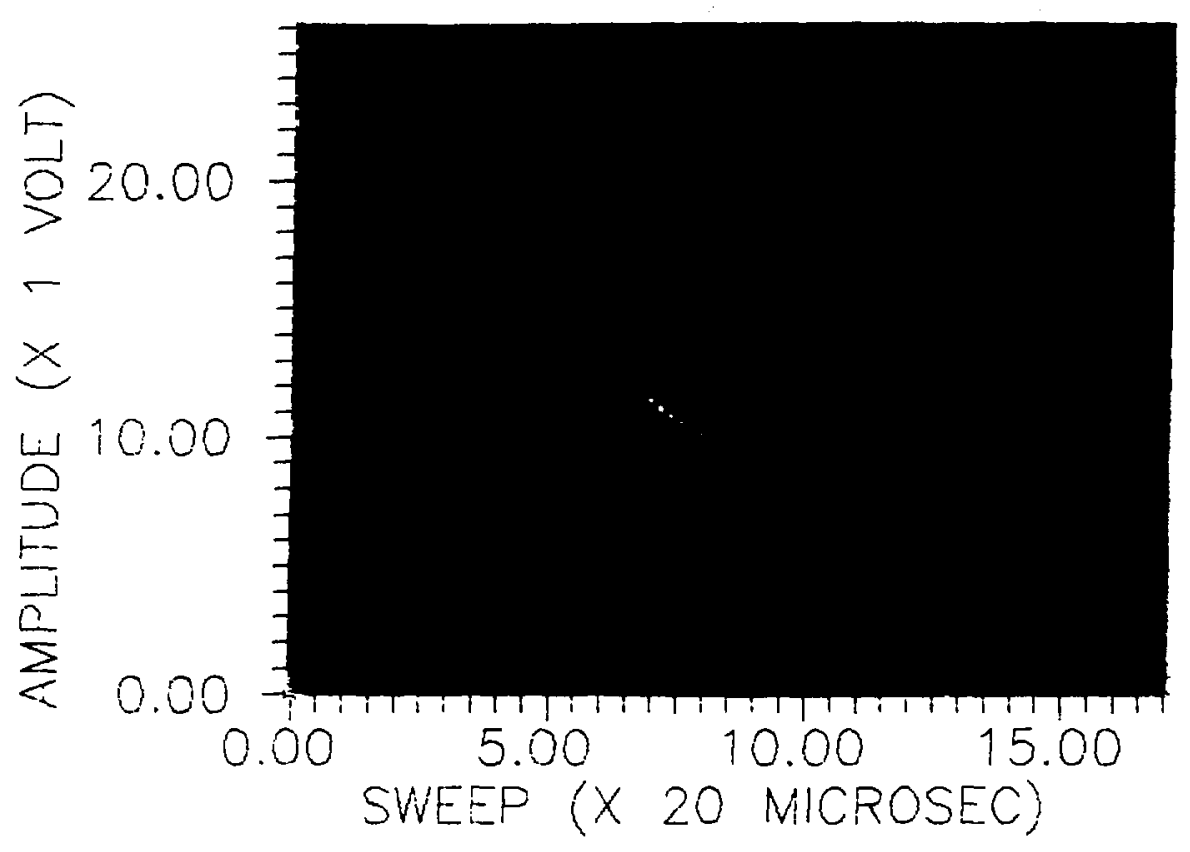

Fig.7 Overload Resizonse Comparison, 20 Microsec Sweep Multiplier. 


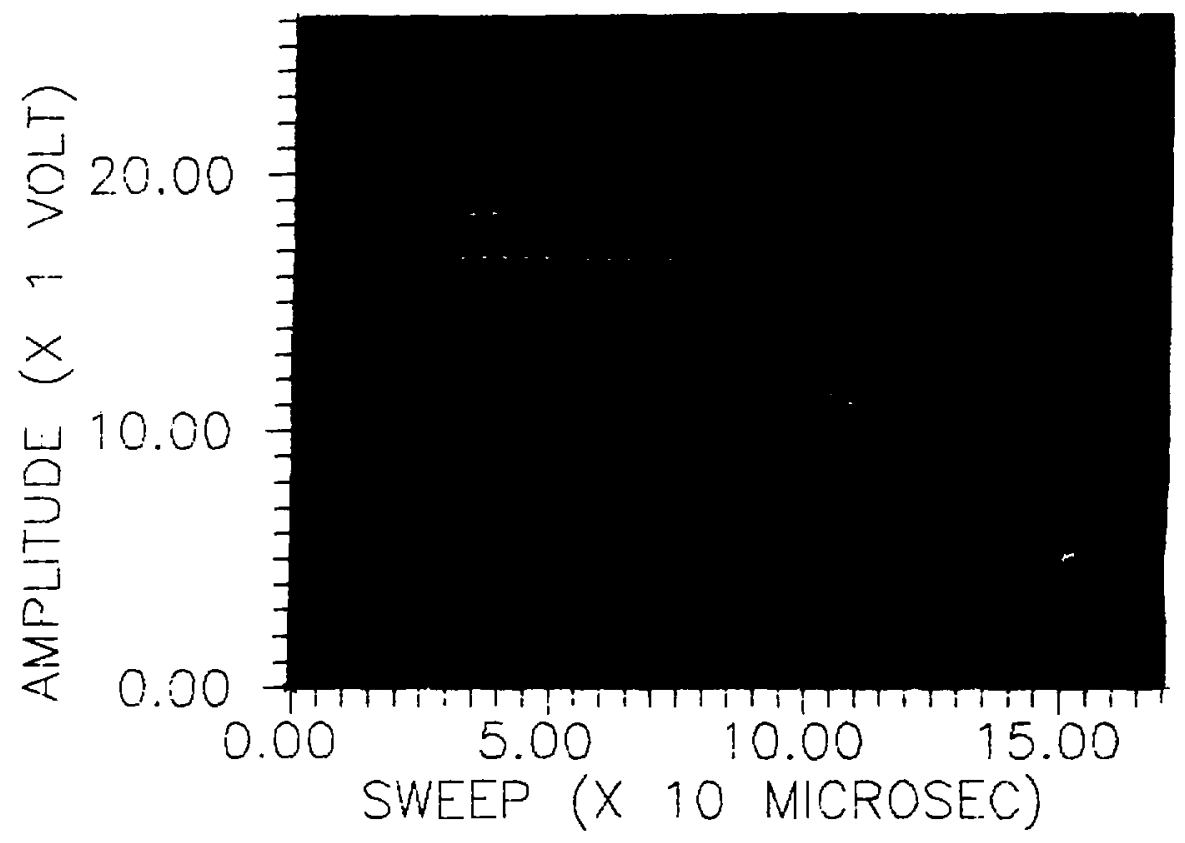

Fig. 8 Overload Response Comparison, 10 Microsec Sweep Multiplier. 
dimensions). The collection time "blip" is clearly visible in either amplifier. In principle, the Filter Amplifier output should drop quickly to baseline (the data pulse time constant is only two microseconds) and remain at baseline until positive ion collection. At this time, the abrupt departure of the true induction effect, which has reached its limiting value, from the compensation of the filter system will initiate a negative-going transient which then decays back to baseline with a mix of time constants of the filter system.

Figures 5 through 8 provide additional comparisons under the same conditions as for Fig. 4 except for successive increases in horizontal sweep speed. The Filter Amplifier is not ideal since a small variation in baseline can be seen in each of these figures. These residual variations imply corresponding discrepancies between the filter, as implemented, and the true induction effect.

It is not necessary to focus only upon the overload response to observe the effect of slow-induction filtering. Figs. 9 and 10 were taken at a low value of voltage ( 3200 volts) such that relatively few overloaded pulses were able to occur. The vertical scope gain was set at .5 volts/cm and internal scope triggering was allowed. Traces from each amplifier are displayed, these were photographed under equal film exposure time and scope brightness. In each figure, data pulses may be observed initially, the data pulse time constant was maintained at 2 microseconds. By comparing results for the two amplifiers, one observes that the Filter Amplifier data pulse exhibits less broadening and a more rapid return to baseline than for the Reference Amplifier. The improved data pulse shape for the Filter Amplifier can be useful in reducing pileup distortion, even when overloading does not occur.

One additional test was carried out, and the results are shown in Figs. 11 and 12. Sixty cycle/sec test pulses were fed to the preamplifier and pulse-height-analyzed by both amplifiers in the presence of overloading at the higher voltage settings. Again, as in all of the other comparisons, amplifier gains were adjusted to the same value. For pulse amplitude analysis the baseline level was set to zero volts for both amplifiers using DC level shifters in the pulse analysis system. A coincidence system was used to enable the pulse height analyzer only for the test pulse. Although test pulses occur at fixed time intervals, the ionizing events are at random and 


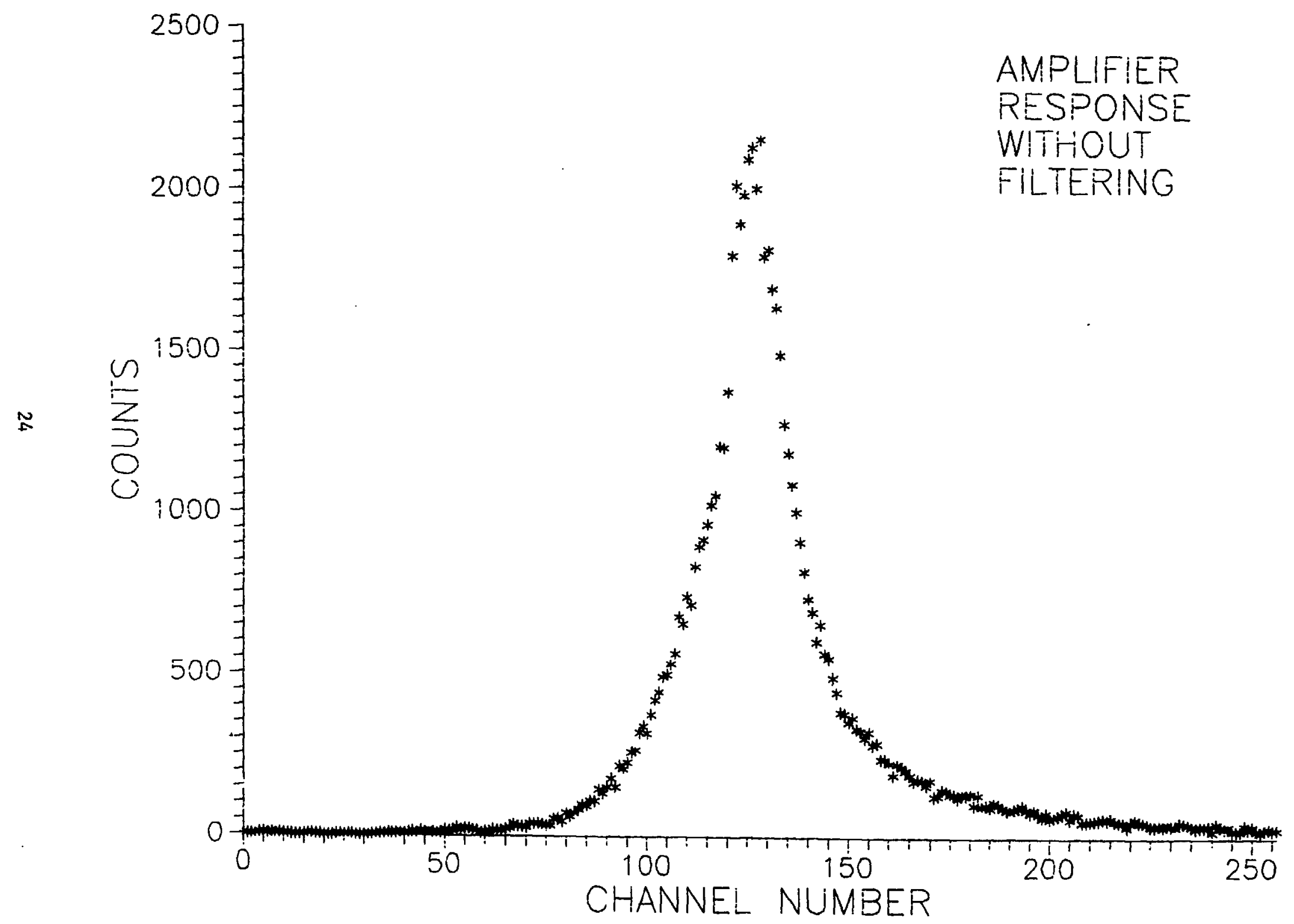

Fig.9 Reference Amplifier Pulse Response. Low Overload Counting. 


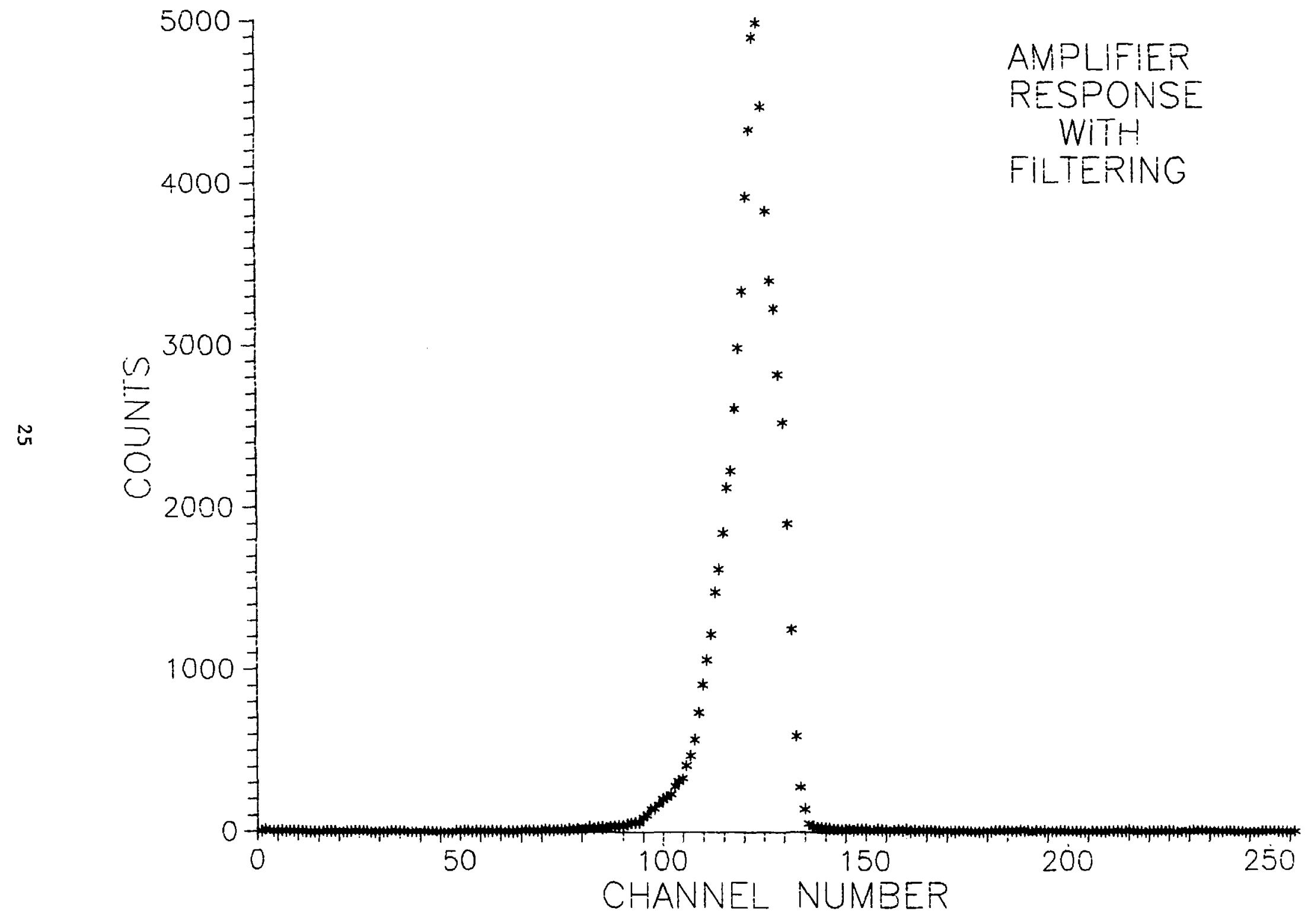

Fig. 10

Filter Amplifier Pulse Response, 


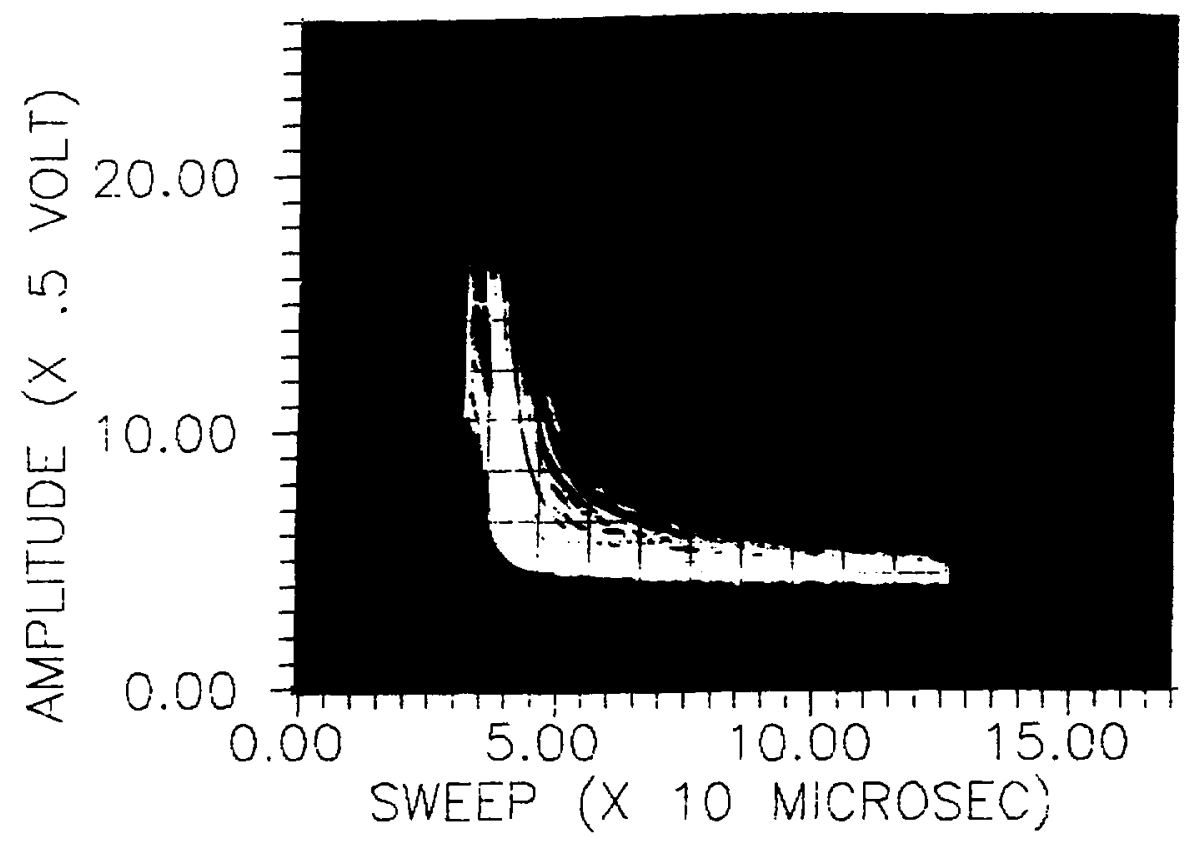

Fig.11 Reference Amplifier Test Pulse Spectrum At High Overload Rates. 


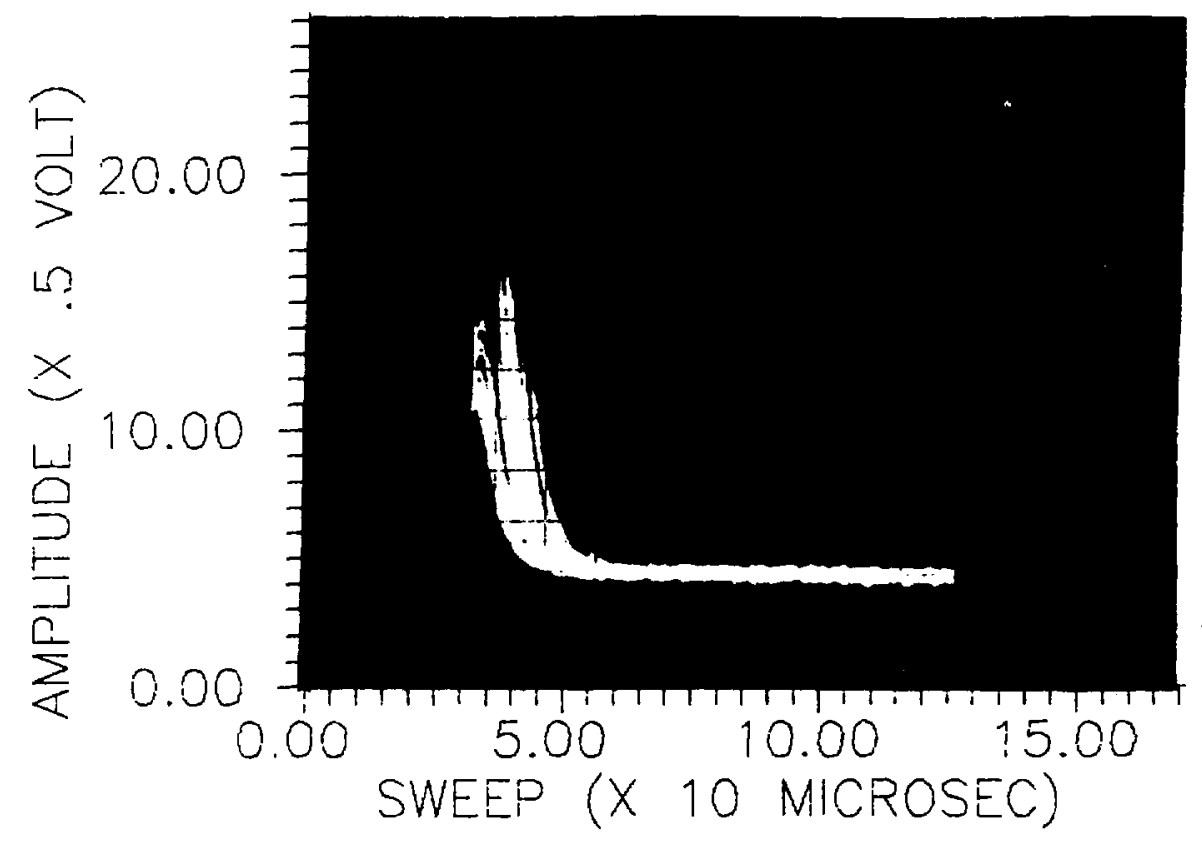

Fig.12 Filter Amplifier Test Pulse Spectrum At High Overload Rates. 
consequently a pulse height spectrum indicates the degree to which distortion occurs from perturbations to the baseline which exist when a test pulse is generated. Data for each spectrum was accumulated for the same time period. In the absence of ionizing events, test pulse peaks from both amplifiers occurred at channel 122 and distributions at half maximum were about two channels wide.

As can be seen from a comparison of the two spectra, lack of slow component filtering can introduce large spectroscopic distortion into data taken in the presence of overloads. The result from the Reference Amplifier (Fig. 11) shows scattering of events over almost the entire spectrum together with a badly degraded line shape. The observed broadening of the Filter Amplifier distribution could be further reduced as the filter approximation is improved to agree better with the true induction profile. Some residual degradation at the low amplitude side of the spectrum will always remain due to the failure of the simple pole-zero approximation at ion collection time. The test pulse line from the Filter Amplifier (Fig. 12) has been broadened but to a much lesser extent that for the Reference Amplifier and there are few events far-removed from the main peak.

\section{CONCLUSIONS}

Although the ideal filter for the proportional counter extended induction effect cannot be precisely realized due to the discontinuous nature of the induction effect at ion collection time, a substantial improvement in the quality of spectroscopy can be brought about using a set of only three polezero filters. These must be tuned to the positive ion collection time for each counter. This collection time is sensitive to all of the parameters of the counter and especially to the ratio of cathode diameter to anode diameter.

With the appropriate filter installed, however, distortion of pulse amplitude spectra due to overloading and high count rates will be significantly lower than for conventional amplifiers which do not contain a filter even if baseline restoration features are incorporated into the amplifier design. 


\section{REFERENCES}

1. J.M. Larson and J.E. Powell, "Spectrum Distortion from Amplifier Overloads in Proton Recoil Proportional Counting," Nucl. Inst. Methods, 71, p. 157 (1969).

2. D.H. Wilkinson, "Ionization Chambers and Counters," Cambridge University Press (1950).

3. E. Fairstein and J. Hahn, "Nuclear Pulse Amplifiers-Fundamentals and Design Practice," Nucleonics, p. 56 (July 1965).

4. J.L. Blankenship and C.H. Nowl in, "New Concepts in Nuclear Pulse Amplifier Design," IEEE Transactions of Nuclear Science, p. 495 (June 1966).

5. Wai-Kai Chen, "Passive and Active Filters," John Wiley and Sons (1986).

6. W.C. Davidon, "Variable Metric Method for Minimization," ANL-5990 (1959).

7. J.M. Larson, "A Wide-Band Charge Sensitive Preamplifier for Proton Recoil Proportional Counting," ANL-7517 (1969). 


\section{DISTRIBUTION LIST FOR ANL/FPP-TM-234}

\section{Internal:}

E. Bennett (15)
C. Baker
Y. Gohar
R. Mattas
R. MoKnight
K. Porges

D. Smith

FPP Files (15)

ANL Contract File

ANL Libraries

ANL Patent Dept.

TIS Files ( 3 )

\section{External:}

DOE-OSTI, for distribution per UC-420 (37)

Manager, DOE-CH

M.A. Abdou, University of California, Los Angeles

T. Alsmiller, Oak Ridge National Laboratory

E. Arthur, Los Alamos National Laboratory

M. Baht, BNL

J. Barnes, Oak Ridge National Laboratory

S. Berk, US Department of Energy

L. Berry, Oak Ridge National Laboratory

D. Berwald, Grumman

R. Brogli, EIR

F. Carre, Centre D'etudes Nucleaires de Saclay

G. Casini, Joint Research Centre

E. Cheng, GA Technologies

J. Clarke, US Department of Energy

R. Conn, University of California, Los Angeles

W. Davidson, Los Alamos National Laboratory

A. Davies, US Department of Energy

R. Dowling, US Department of Energy

Du Shu-Hua, Southwest Institute of Nuclear Physics and Chemistry

D. Dudziak, Los Alamos National Laboratory

D.V. Efremov, SRIEA

M. Embrechts, Rensselaer Polytechnic Institute

A.M. Epinatiev, IET

U. Fischer, KfK

C. Fu, Oak Ridge National Laboratory

T. Fuketa, JAERI

T. Gabriel, Oak Ridge National Laboratory

L. Green, Westinghouse Hanford Company

E. Greenspan, AEC

C. Gung, University of California, Los Angeles

G. Haas, US Department of Energy

L. Hansen, Lawrence Livermore National Laboratory

P. Heming, US Department of Energy

N. Hertel, University of Texas

R.J. Howerton, Lawrence Livermore National Laboratory

J.H. Huang, Southwest Institute of Nuclear Physics and Chemistry

T. Iguchi, University of Tokyo

Y. Ikeda, JAERI 
Y. Ishiguro, JAERI

Jian Wen-Mian, Southwest Institute of Nuclear Physics and Chemistry

I. Jun, University of California, Los Angeles

Y. Kaneko, JAERI

B.G. Karasev, SRIEA

W. Kastenberg, University of California, Los Angeles

G. Kessler, KfK

I. Kimura, Kyoto University

C. Konno, JAERI

T. Kosako, JAERI

L.P. Ku, Princeton Plasma Physics Laboratory

M. Kuchle, KfK

A. Kumar, University of California, Los Angeles

I.V. Kurchatov, IAE

R. Labauve, Los Alamos National Laboratory

I.V. Lavrentjev, SRIEA

J.D. Lee, Lawrence Livermore National Laboratory

Liu Cheng-An, Southwest Institute of Nuclear Physics and Chemistry

H. Maekawa, JAERI

K. Maki, JAERI

J. Maniscalco, TRW

F. Mann, Hanford Engineering Development Laboratory

D. Matthews, GA Technologies

C. Maynard, University of Wisconsin-Madison

R. McFarlane, Los Alamos National Laboratory

K. Miya, University of Tokyo

R. Moir, Lawrence Livermore National Laboratory

S. Mori, JAERI

T. Mori, JAERI

D. Muir, Los Alamos National Laboratory

M. Nakagawa, JAERI

H. Nakajima, Kyushu University

T. Nakamura, JAERI

M. Nakazawa, University of Tokyo

Y. Oka, University of Tokyo

P.V.V. Orlov, IAE

Y. Oyama, JAERI

S. Pearlstein, BNL

R. Peelle, Oak Ridge National Laboratory

S. Pelloni, EIR

S.T. Perkins, Lawrence Livermore National Laboratory

G. Pomraning, University of California, Los Angeles

C. Ponti, Joint Research Centre

Qiu Li-Jian, Southwest Institute of Nuclear Physics and Chemistry

M. Ragheb, University of Illinois

G. Reffo, Joint Research Centre

M. Roberts, US Department of Energy

R. Roussin, RSIC

R. Santoro, Oak Ridge National Laboratory

M. Sawan, University of Wisconsin-Madison

J. Schifield, Oak Ridge Natioanl Laboratory

K. Schultz, GA Technologies

J.P. Schwartz, Centre D'etudes Nucleaires de Saclay

Y. Seki, JAERI 
H. Sekimoto, Tokyo Institute of Technology

G. Shatalov, IAE

Shen Ji-An, Southwest Institute of Nuclear Physics and Chemistry

K. Shin, Kyoto University

M. Song, University of California, Los Angeles

W. Stacey, Georgia Tech

D. Steiner, Rensselaer Polytechnic Institute

J. Stepanek, EIR

Yu. Strebkov, IET

K. Sugiyama, Tohoku University

K. Sumita, Osaka University

A. Takahashi, Osaka University

T. Tone, JAERI

K. Tsuda, JAERI

Y. Watanabe, University of California, Los Angeles

R. White, Oak Ridge National Laboratory

R. Whitley, TRW

G. Woodruff, University of Washington

S. Yamaguchi, JAERI

Y. Yamamoto, Osaka University

M. Yoshikawa, JAERI

K. Young, Princeton Plasma Physics Laboratory

P. Young, Los Alamos National Laboratory

M.Z. Youssef, University of California, Los Angeles 